\title{
Photolithographic Properties of Photosensitive Sol-gel Materials and Their Application to Optical Waveguides
}

\author{
Kentarou Tamaki, Tomohiro Utaka, Hideaki Takase, Yuichi Eriyama, and Takashi Ukachi \\ JSR Corporation, Tsukuba Research Laboratories \\ 25 Miyukigaoka, Tsukuba, Ibaraki, 305-0841 Japan \\ telephone: +81-298-56-1109, facsimile: +81-298-56-1504 \\ kentarou_tamaki@jsr.co.jp
}

\begin{abstract}
Novel photosensitive sol-gel materials, which are comprised of siloxane oligomers and a photo acid generator (PAG), have been developed. The spectroscopic studies led to the observation that sol-gel reaction was affected by a balance of between PAG and amine content added as catalysts. Additionally, the balance strongly influenced the line pattern profile formed with exposure, which indicated that the photolithographic properties changed from negative to positive tone with the amine content. An optical channel waveguide based on sol-gel materials was fabricated through photolithographic processing and showed low propagation losses that were $0.3 \mathrm{~dB} / \mathrm{cm}$ and $0.6 \mathrm{~dB} / \mathrm{cm}$ at $1.31 \mu \mathrm{m}$ and $1.55 \mu \mathrm{m}$, respectively, in addition to excellent long-term reliability.

Keywords: Photosensitive sol-gel, photolithography, Channel Waveguides
\end{abstract}

\section{Introduction}

Sol-gel processing offers a low-temperature synthetic route, and compositional and microstructural control [1]. The processing provides new materials as well, i.e. inorganic-organic hybrids, in which an inorganic network structure is organically modified at the molecular level, leading to several applications, especially in recent years, to optical and electronic applications. As another feature of sol-gel materials, the introduction of organic groups into an inorganic network improves mechanical properties and makes thick film or coating production possible. Silicates have been extensively investigated as sol-gel materials. Silicon alkoxides as sols form polysilicate gels through hydrolysis and condensation under either acidic or basic catalyst [1].

Photo-reactive groups have been introduced into sol-gel materials to create a photo-patternable properties. Several researchers have already reported photolithographic properties in the photoresist, and optical waveguides area [2-4]. The photo-patternable sol-gel materials showed an attractive photolithographic property, but still may be accompanied by shrinkage, cracks on thick films or sometimes phase separation. The sol-gel materials, however, exhibit high thermal stability and in most cases high transparency, so the application to optical planar waveguides has been extensively investigated.

Recent rapid growth of optical telecommunication networks requires us to develop optical components and devices. Inorganic materials such as silica-based compounds have been widely developed as conventional optical planar waveguides. Their fabrication processing requires lots of time, cost and many steps such as a chemical vapor deposition (CVD) or a flame hydrolysis deposition (FHD). Currently, several kinds of polymeric and sol-gel materials, which have less intrinsic absorption in the near-ir wavelength region, have been developed and introduced to fabricate the waveguides by using the spin-coating technique instead of the CVD or FHD processes [5]. Although polymeric materials have shown advantages in making the process simple, in many cases, it is still required to employ a reactive ion etching (RIE) technique to make fine waveguide 
patterns [6]. It is expected that materials will be developed to allow us to make fine patterns through photolithographic technique without RIE.

In this paper, we discuss the reaction mechanism of new photosensitive sol-gel materials and their unique photolithographic properties that are controllable by amine compound addition. We also demonstrate an optical waveguide fabricated using the materials through photolithographic technique.

\section{Experimental}

\section{2-1. Materials}

Photosensitive sol-gel materials we prepared for this study consist of organo-functional siloxane oligomers, $0.1 \mathrm{wt} \%$ photo acid generator (PAG), propylene glycol monomethyl ether as a solvent, and in some cases, an amine compound as an acid quencher. The siloxane oligomers were synthesized through hydrolysis and condensation of alkyl- and/or aryl- alkoxysilanes according to the procedure described in ref. [7].

For waveguide fabrication, PJ5007 and PJ5010 were used as a core material and a cladding material, respectively. Both of them also consist of the siloxane oligomers and a PAG, and were modified by changing the oligomer structures to control refractive index. The refractive index of the cured core material (PJ5007) was designed to be 1.469 , which is $0.4 \%$ higher than that of the cured cladding material (PJ5010). In addition, an amount of the amine contained in PJ5007 was optimized to obtain a good line pattern shape. Both materials were diluted with $45 \mathrm{wt} \%$ propylene glycol monomethyl ether.

\section{2-2. Sol-gel reaction}

Infrared (IR) spectra were measured to monitor the sol-gel reaction and were obtained with a BIO-RAD FTS-6000 FT-IR spectrometer at a resolution of $4 \mathrm{~cm}^{-1}$. The sample was coated at a thickness of $2 \mu \mathrm{m}$ onto a silicon substrate. IR spectra were measured after each process described in Figure 1.

\section{2-3. Photolithographic properties}

Photolithographic property was evaluated with a line pattern formed through photolithographic processes (Figure 1) that are common steps for microlithography using a photoresist. The sample was prepared at a thickness of $10 \mu \mathrm{m}$ on a silicon substrate by spin-coating and prebaked at $100{ }^{\circ} \mathrm{C}$ for 1 minute. The sample was exposed to a $365 \mathrm{~nm}$

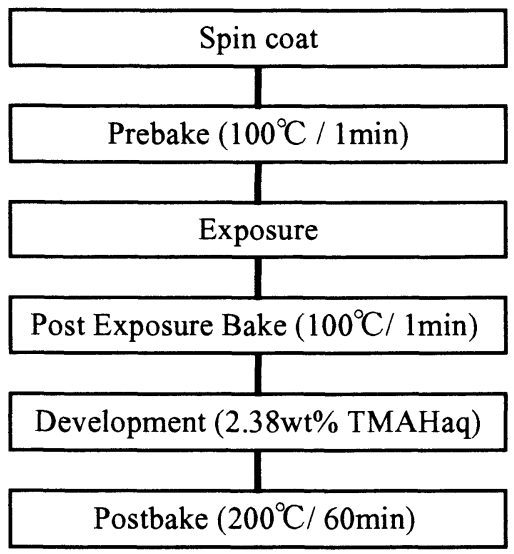

Figure 1. Sample preparation procedure for photolithography property.

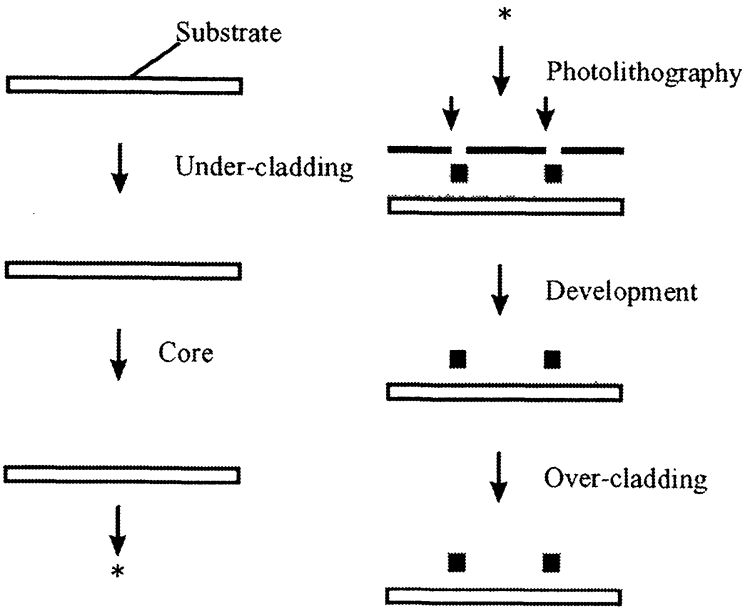

Figure 2. Schematic diagram of fabrication process for optical channel waveguides.

UV light (Mask Aligner PLA-501F made by CANON) at $100 \mathrm{~mJ} / \mathrm{cm}^{2}$ through a photomask with a $10 \mu \mathrm{m}$ line and $30 \mu \mathrm{m}$ space. After exposure, the sample was baked at $100{ }^{\circ} \mathrm{C}$ for 1 minute and developed with $2.38 \mathrm{wt} \%$ tetramethyl ammonium hydroxide (TMAH) aqueous solution. After postbaking at $200{ }^{\circ} \mathrm{C}$ for 60 minutes, the obtained line pattern was observed with scanning electron microscopy (SEM) to measure the line width.

\section{2-4. Optical waveguide fabrication}

A channel type straight waveguide was fabricated using PJ5007 and PJ5010 according to the process in Figure 2. PJ5010 as an under-cladding layer was spin-coated onto a silicon substrate and prebaked at $120^{\circ} \mathrm{C}$ for 10 minutes to 
evaporate the solvent. The sample was exposed to UV light at $500 \mathrm{~mJ} / \mathrm{cm}^{2}$ and postbaked at $200^{\circ} \mathrm{C}$ for 60 minutes to complete sol-gel reaction. PJ5007 was coated onto the cladding layer to form a core and was then prebaked. Exposure was carried out using the photomask in the previous section. A so-called post exposure bake (PEB) was performed at $100{ }^{\circ} \mathrm{C}$ for 1 minute, and it was developed with TMAH solution. Finally, the fabricated sample was covered with PJ5010 in the same manner as the under-cladding formation.

\section{2-5. Waveguide characteristics}

The optical propagation losses of the fabricated waveguide were evaluated with a function of wavelength by using a photo spectrum analyzer equipped with an optical core pitch measuring system. Furthermore, the propagation losses at both $1.31 \mu \mathrm{m}$ and $1.55 \mu \mathrm{m}$, which are available for telecommunication transmission, were measured by a cut-back method.

In order to evaluate the long-term reliability of the waveguides, a difference in refractive indices between core and cladding materials $(\Delta \mathrm{n})$ was measured using a Prism Coupler (METRICON Model2010) instead of monitoring change in the optical losses. The samples were kept in an oven at $85{ }^{\circ} \mathrm{C}$ and $85 \%$ R.H., and the refractive index of each sample on the substrate was measured at room temperature.

\section{Results and Discussion}

\section{3-1. Sol-gel reaction mechanism}

For the sample containing $0.1 \mathrm{wt} \%$ PAG without amine, IR spectrum showed a broad peak around $3,300 \mathrm{~cm}^{-1}$ which corresponds with the

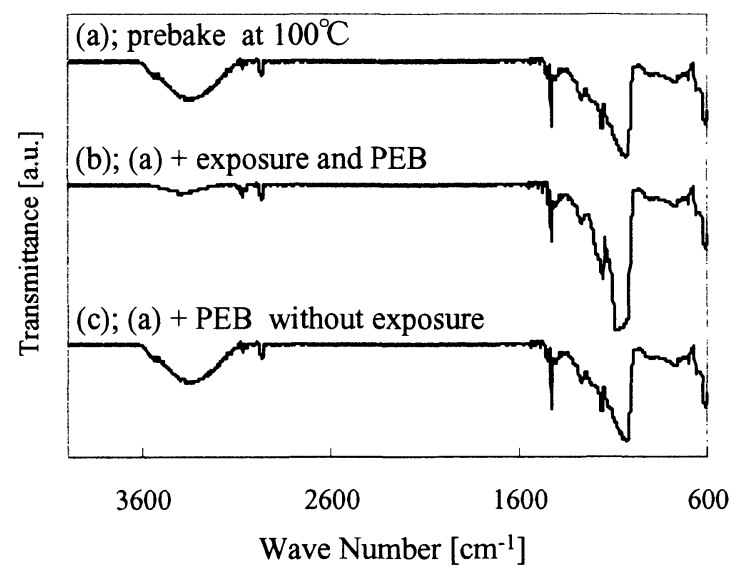

Figure 3. IR spectra of the sol-gel material with 0.1 wt $\%$ PAG. hydroxyl groups, indicating that the siloxane oligomers include silanol groups (Si-OH) generated by hydrolysis of alkoxyl groups. The silanol groups were still observed even after prebaking at $100{ }^{\circ} \mathrm{C}$ (Figure 3(a)). The peak strength decreases significantly with exposure accompanied by an increase in the peak at 1,100 $\mathrm{cm}^{-1}$ assigned to the siloxane structure (Si-O-Si) (Figure 3(b)). It is supposed that silanol group condensation proceeds under the acid generated with exposure. On the other hand, the spectrum after prebaking and that without exposure seem to be similar. This indicates that condensation does not occur without exposure (Figure 3(c)).

In the presence of an amine compound, prebaking at $100{ }^{\circ} \mathrm{C}$ is sufficient to condense silanol groups although condensation is not observed at $80{ }^{\circ} \mathrm{C}$ in Figure $4(\mathrm{a}, \mathrm{b})$. This indicates that silanol groups undergo condensation under the amine catalyst at high temperature. UV exposure onto the sample after prebaking at $80{ }^{\circ} \mathrm{C}$ does not alter IR absorption, supposing that the acid generated with exposure is neutralized by the amine (Figure 4(c)). Further baking at $100{ }^{\circ} \mathrm{C}$ without exposure decreases the silanol peak strength and initiates condensation (Figure 4(d)).

It is considered that the siloxane oligomers in the sample form a crosslinked polysiloxane network with different catalytic actions as shown in Figure 5. The efficiency of the acid for condensation of silanol groups seems higher than that of the amine because silanol groups disappear easily under acidic catalyst while high temperature is required under the amine catalyst. The amine acts not only as an acid quencher but also as a catalyst.

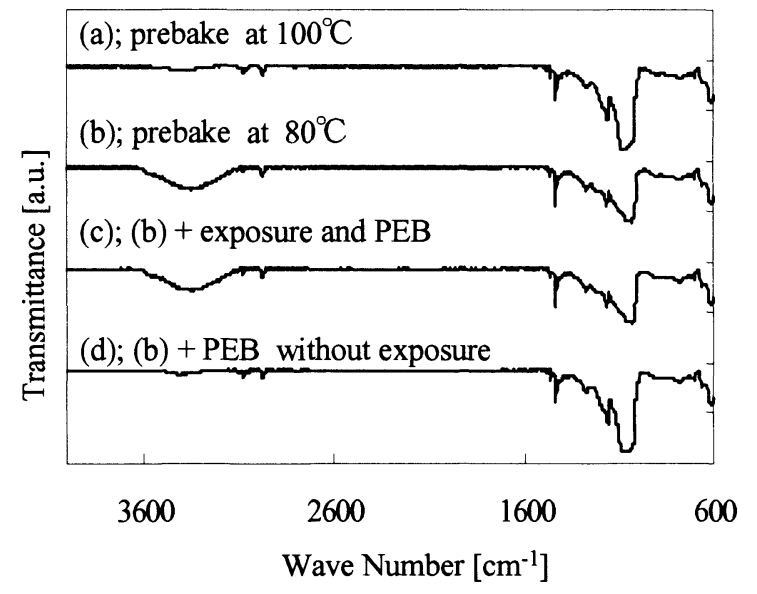

Figure 4. IR spectra of the sol-gel material with 0.1 $w t \%$ PAG and $0.025 \mathrm{wt} \%$ amine. 


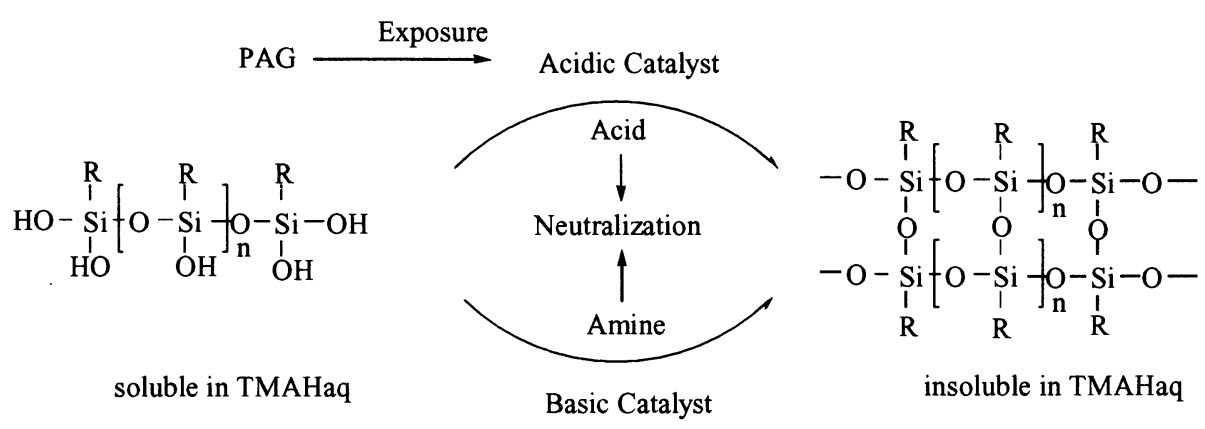

Figure 5. Reaction mechanism of photosensitive sol-gel materials.

Table 1. Photolithography type of sol-gel materials containing different amount of amine

\begin{tabular}{cccc}
\hline Amine $/ \mathrm{wt} \%$ & Type of photolithography & Exposed area & Unexposed area \\
\hline $0 \sim 0.015$ & negative tone & insoluble & soluble \\
0.020 & - & soluble & soluble \\
$0.025 \sim 0.030$ & positive tone & soluble & insoluble \\
\hline
\end{tabular}

\section{3-2 Photolithographic properties}

Most chemically amplified resists contain acid quenchers such as amine compounds to control a pattern profile. The quenchers play an important role in improving critical dimension controllability because they quench the acid diffused into an unexposed area.

Addition of the amine compound influences the photolithographic property is shown in Table 1. The exposed area was cured by the acid generated with exposure when the amine was increased to $0.015 \mathrm{wt} \%$. The area was insoluble by any solvent, a so-called "negative tone". On the other hand, the unexposed area was developed completely with a TMAH solution which indicates no condensation occurres in the unexposed area. With the addition of $0.02 \mathrm{wt} \%$ amine, both exposed and unexposed areas dissolved into a TMAH solution. This indicates that the added amine is enough to neutralize the generated acid and to inhibit condensation. Amine in excess of $0.025 \mathrm{wt} \%$ acted as a basic catalyst and could initiate condensation at high temperature without exposure. The unexposed area became insoluble due to condensation catalyzed by the amine. In the exposed area, a certain amount of amine was neutralized with the generated acid, and the residual amine was not enough to start condensation. In this case, the photolithography implies "positive tone". Figure 6 shows the effect of amine content on the line width. The line pattern becomes narrower with the addition of the amine. The line exhibits an identical profile at $0.01 \mathrm{wt} \%$ amine as shown in Figure 7. At $0.02 \mathrm{wt} \%$ amine, the exposed area as well as the unexposed area were completely soluble. With the addition of more than $0.02 \mathrm{wt} \%$ amine, the unexposed area, the size of which was $30 \mu \mathrm{m}$, became insoluble, and finally, all areas became insoluble. It is noteworthy that the photosensitive sol-gel materials used here can convert photolithographic type by controlling the amount of amine.

It is well-known that exposure dose affects the line pattern shape of the materials. In a series of the sample containing less than $0.02 \mathrm{wt} \%$ amine, the materials showed negative tone, and the sensitivity was $55 \mathrm{~mJ} / \mathrm{cm}^{2}$ for the formulation without amine (Figure 8). On the other hand, the sample containing $0.025 \mathrm{wt} \%$ amine was insoluble at lower doses than $100 \mathrm{~mJ} / \mathrm{cm}^{2}$. The phenomenon is considered to be a situation in which an excess of amine catalyzed condensation



Figure 6. Relationship between line width and amine content for the sol-gel materials with $0.1 \mathrm{wt} \%$ PAG at the exposure dose of $100 \mathrm{~mJ} / \mathrm{cm}^{2}$. 


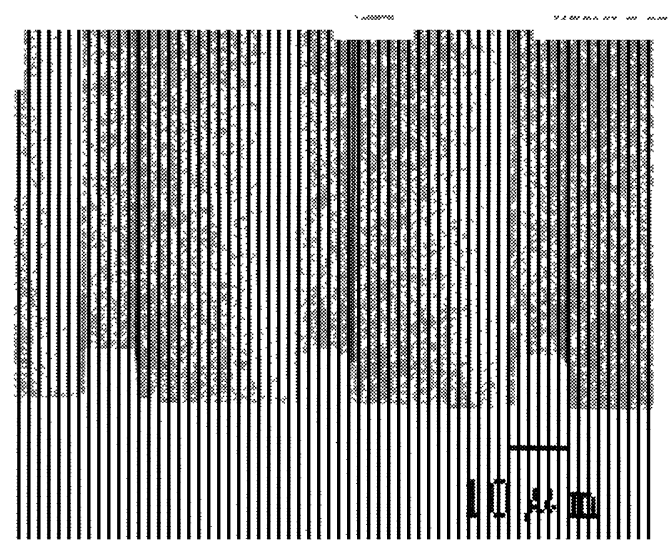

Figure 7. Cross sectional SEM image of line pattern profile using negative type sol-gel materials with $0.01 \mathrm{wt} \%$ amine.

of silanol groups. The added amine seems to be completely neutralized with acid generated at exposure between 100 and $150 \mathrm{~mJ} / \mathrm{cm}^{2}$. It implies "positive tone" in the range of the doses. Since further exposure to more than $150 \mathrm{~mJ} / \mathrm{cm}^{2}$ generates much more acid than the added amine, the exposed area on the sample is cured to work as so-called "negative tone". The sensitivity curve of the sample with $0.025 \mathrm{wt} \%$ amine exhibits high solubility contrast. It gives a fine line pattern under positive tone (Figure 9).

\section{3-3 Optical waveguide fabrication}

A cross-sectional microscopic photograph of a channel type optical waveguide fabricated with PJ5007/PJ5010 shows a square core with $10 \mu \mathrm{m} \mathrm{x}$ $10 \mu \mathrm{m}$ (Figure 10). It is also observed that the core is surrounded with cladding layers.

\section{3-4 Waveguide characteristics}

Optical loss spectrum of the fabricated waveguide with $1 \mathrm{~cm}$ core length was measured and shown in Figure 11. The characteristic absorption peaks are observed at 1.13, 1.17, 1.39 and $1.64 \mu \mathrm{m}$. It is considered that these peaks are assigned to the absorption of $\mathrm{C}-\mathrm{H}$ and $\mathrm{O}-\mathrm{H}$ overtone stretches and combinations of overtone with deformation. These peaks raise the optical losses, nevertheless, the contribution seems to be minor at $1.33 \mu \mathrm{m}$ and $1.55 \mu \mathrm{m}$, which are used for telecommunication. These propagation losses were $0.3 \mathrm{~dB} / \mathrm{cm}$ and $0.6 \mathrm{~dB} / \mathrm{cm}$ at each wavelength, respectively. The measurement was carried out using the cut-back method which could remove the coupling loss between the core and the fiber. Besides, it is noteworthy that the polarization

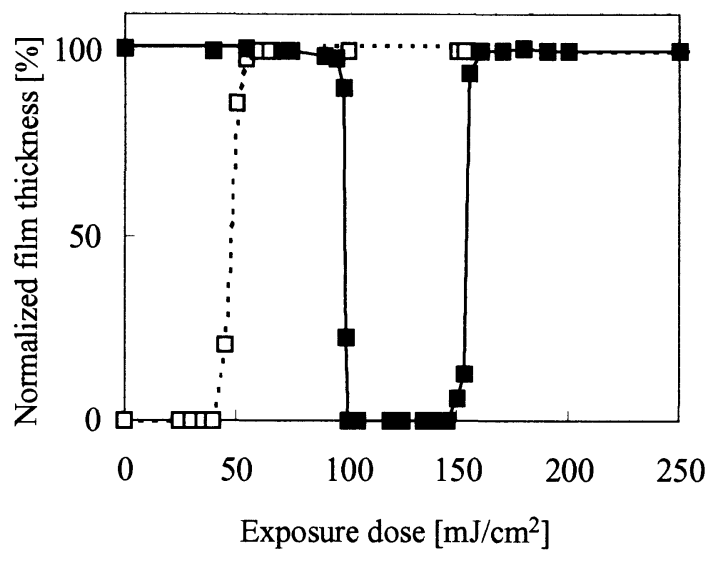

Figure 8. Sensitivity curves for the sol-gel materials without amine ( $\square$ ) and with $0.025 \mathrm{wt} \%$ amine ( $\square$ ).

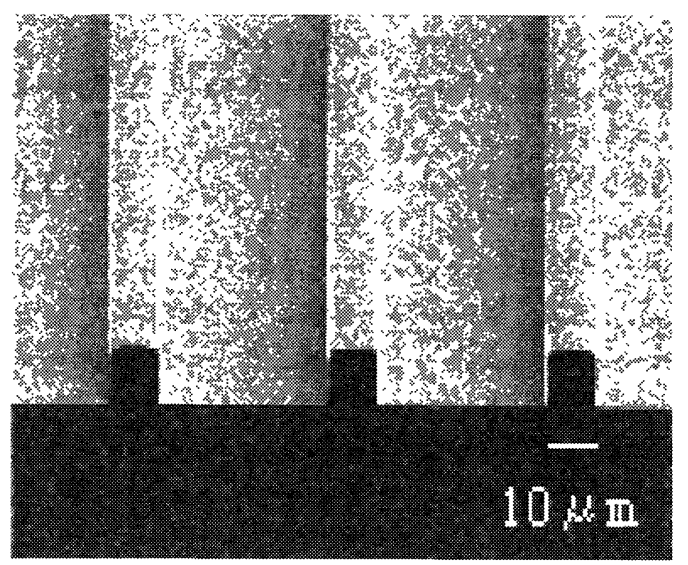

Figure 9. Cross sectional SEM image of line pattern profile of the positive type sol-gel materials with $0.025 \mathrm{wt} \%$ amine.

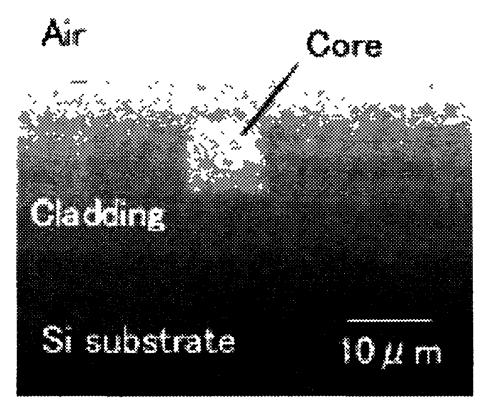

Figure 10. Microscopic photograph of cross section of channel waveguide fabricated with PJ5007/5010. 
dependent losses were not greater than $0.1 \mathrm{~dB} / \mathrm{cm}$. The details will be discussed elsewhere.

Figure 12 shows $\Delta \mathrm{n}$ change calculated from the refractive indices of both samples. The $\Delta \mathrm{n}$, which is an important required characteristic for optical waveguides, shows stability even after a 4,000 hour high-temperature high-humiding environmental test.

\section{Conclusions}

Novel photolithographic sol-gel materials including siloxane based oligomers and a photoacid generator have been developed. IR measurement showed that condensation of silanol groups to form the crosslinked gels could be controlled by a balance of between photochemically generated acid and the amine contents. The photolithographic property was strongly affected by the amount of both the acid and the amine. The formation tone depended on the amine content. A channel type optical waveguide using sol-gel materials was demonstrated through photolithographic process. SEM photograph showed the waveguide with a good core shape. The fabricated waveguide indicated low propagation losses at telecommunication transmittance, i.e. $0.3 \mathrm{~dB} / \mathrm{cm}$ and $0.6 \mathrm{~dB} / \mathrm{cm}$ at $1.33 \mu \mathrm{m}$ and $1.51 \mu \mathrm{m}$, respectively. Furthermore, $\Delta \mathrm{n}$ of the waveguides was definitely stable under long-term high temperature and high humid conditions. Thus, the photosensitive sol-gel materials were considered to have an attractive characteristic for optical waveguide and to be able to make the fabrication process simple.

\section{References}

[1] J. Brinker and G. W. Schener, "SOL-GEL SCIENCE: The Physics and Chemistry of Sol-Gel Processing" Academic Press, Inc. (1990).

[2] M. Sakata, T. Ito, M. Kosuge and Y. Yamashita, J. Photopolym. Sci. Technol., 5, 181 (1992).

[3] K. Suzuki, M. Yamashita, N. Kawakami and A. Nakaue, Jpn. J. Appl. Phys., 35, 6517 (1996).

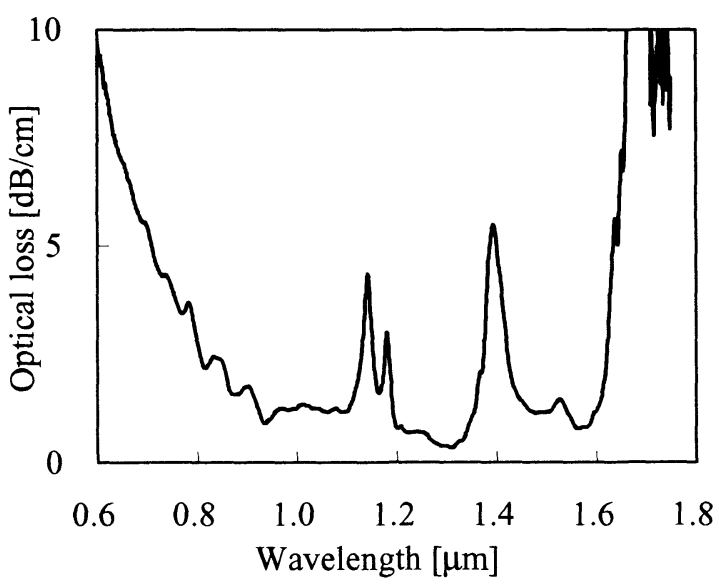

Figure 11. Loss spectrum of the optical channel waveguide fabricated with PJ5007/PJ5010.

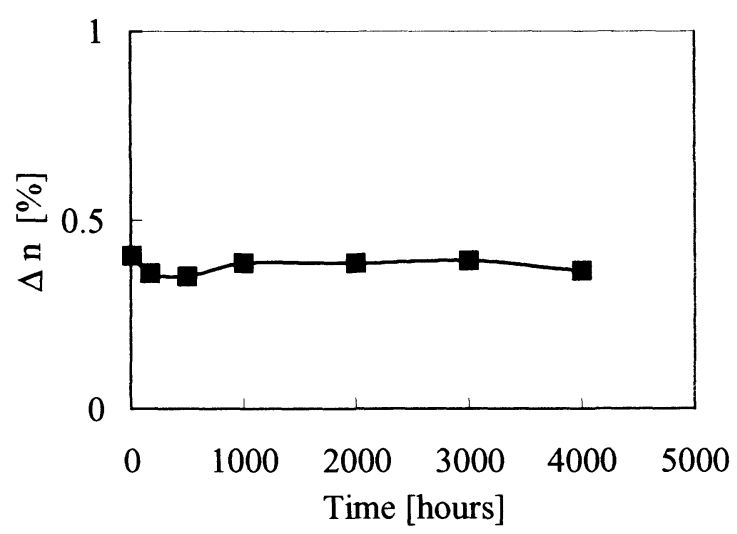

Figure 12. $\Delta \mathrm{n}$ change in refractive indices of PJ5007/PJ5010.

[4] N. Yamada, I. Yoshinaga and S. Katayama, J.Appl. Phys., 85, 2423 (1999).

[5] L. A. Hornak, "Polymer for Lightwave and Integrated Optics: Technology and Applications", Marcel Dekker Inc. (1992).

[6] T. Matsuura, S. Ando, S. Matsui, S. Sasaki and F. Yamamoto, Electron. Lett., 27, 2107 (1993).

[7] JP kokai, 2001-288364. 